\title{
Linear Algebra Based Generalization of the Kennelly's Theorem
}

\author{
Ali Krim*, Abderrazak Lakrim, Driss Tahri \\ Electrical Department, Faculty of Sciences and Technologies, Sidi Mohamed Ben Abdellah University, Morocco
}

Copyright $\mathrm{C} 2019$ by authors, all rights reserved. Authors agree that this article remains permanently open access under the terms of the Creative Commons Attribution License 4.0 International License

\begin{abstract}
The Kennelly theorem which is widely used in three phase systems allows for the delta-star and star-delta conversion and simplification of several electronic circuits. In the present work, we propose a generalization based on the theorem of superposition and some results of linear algebra. Our demonstration is inspired from the proof of the classical Kennelly's theorem. The proposed formulas are very clear and simple. This will make it possible to convert polygon-start and star -polygon if the number of impedances is odd, greater than or equals three. The advantage of our proposal is that it could be understood and programmed easily by undergraduate student when compared to other methods based on the graph theory, which focuses mainly on the mesh-star conversion, which is not possible in all configurations in both ways. This result can be applied to reduce the number of nodes in circuit type models of electrical components and electronic circuits. Thus, the simulation time is reduced.
\end{abstract}

Keywords Delta-star Transformation, Polygon-star Transformation, Distribution Network, Circuit Theory

\section{Introduction}

Thanks to the established electrical laws such as Kirchhoff's laws and Ohm's law, the electrical circuits and systems can be modeled and simulated by using circuit-type simulators. The integration of the original models in these platforms leads in many cases to a complex circuit with a huge number of nodes. Therefore, it is very interesting to find a way to simplify these models.

Several electricity theorems have been developed or generalized to simplify electrical circuits. For example in [1], the author proposes an extension of Kennelly and Thévénin theorem for active networks with three terminals. Also a generalization of Thévénin theorem was proposed in [2]. These theorems are very important for complex circuit analysis [3]. Indeed, the computational time of a simulation rises considerably when the number of nodes is increased. Thus, transformation theorems are required to reduce the number of elements in electrical networks. Other techniques based on the algebraic graph theory allow achieving the same objective [4]. As the well-known Kron reduction [5].

Many papers studied the mesh- star transformation [6], [7] and [8]. The conditions for which the equivalent mesh network of a star one exists impose constraints on the network elements (Wheatstone relationship) [9], [10].

In this paper, we set out to contribute to another generalization of the Kennelly's theorem, which depend only on the number of nodes in the network. This will make it similar to the classical one, which holds without conditions on the network elements. Our formulation is simple and can be programmed under MATLAB. It could also be used to provide either numerical results or formulas for a given network order by using symbolic calculation.

This paper is organized as follows: - Firstly, we present the traditional form of Kennelly theorem. - Secondly, by demonstration we show the possibility to generalize this theorem. - And finally, we perform two examples to exhibit the effectiveness of our proposal.

\section{Kennelly's Theorem}

The circuit in (figure 1) is equivalent to the circuit in (figure 2) that if the impedances are connected by the following relations:

$$
\begin{gathered}
Z_{1}=\frac{Z_{12} \times Z_{31}}{Z_{12}+Z_{23}+Z_{31}} \\
Z_{2}=\frac{Z_{12} \times Z_{23}}{Z_{12}+Z_{23}+Z_{31}} \\
Z_{3}=\frac{Z_{31} \times Z_{23}}{Z_{12}+Z_{23}+Z_{31}}
\end{gathered}
$$




$$
\begin{gathered}
Z_{12}=\frac{Z_{1} \times Z_{2}+Z_{2} \times Z_{3}+Z_{3} \times Z_{1}}{Z_{3}} \\
Z_{23}=\frac{Z_{1} \times Z_{2}+Z_{2} \times Z_{3}+Z_{3} \times Z_{1}}{Z_{1}} \\
Z_{31}=\frac{Z_{1} \times Z_{2}+Z_{2} \times Z_{3}+Z_{3} \times Z_{1}}{Z_{2}}
\end{gathered}
$$

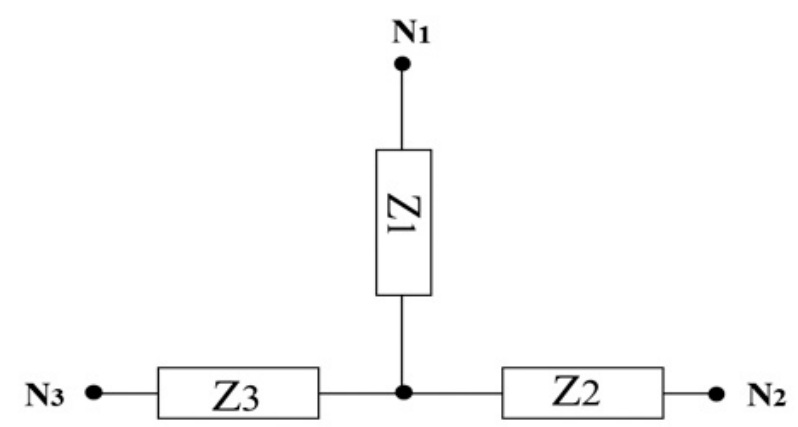

Figure 1. Star network with three nodes

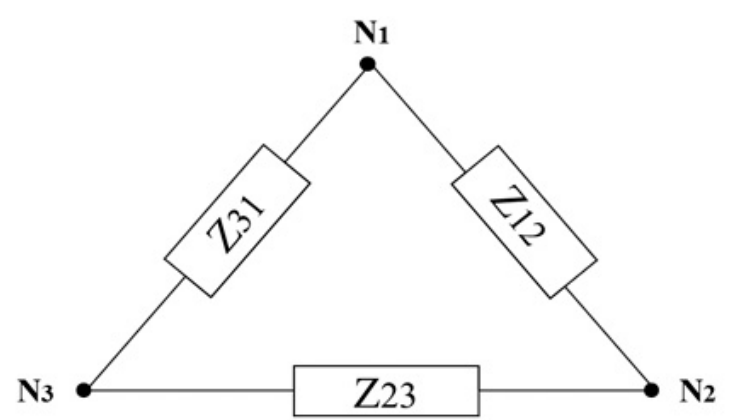

Figure 2. Delta network with three nodes

\section{Generalized Kennelly's Theorem}

The network in polygonal form shown in (figure 3), and the network in star form shown in (figure 4) are equivalent if the number of nodes $\mathrm{Ni}$ is odd and if their admittances are connected by the relation (7) (see (figure 7)) and their impedances are connected by the relation (8) (see (figure 8)).

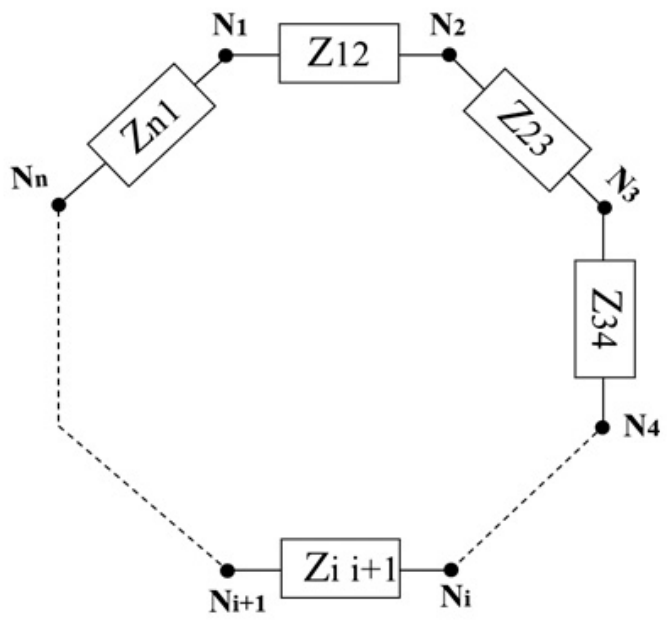

Figure 3. The network in polygonal form

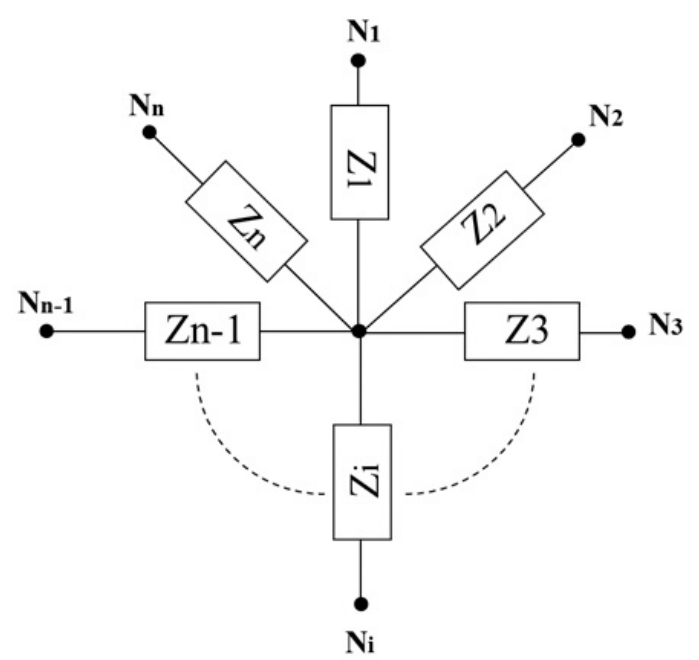

Figure 4. The network in star form

So we have:

$$
Y_{i j}=\frac{1}{Z_{i j}} \text { and } Y_{i}=\frac{1}{Z_{i}}
$$

Equation (7) shows the relationship between the polygonal circuit admittances (Yij) and those of the star circuit (Yi). On the other hand, the equation (8) shows the relationship between the star circuit impedances $(\mathrm{Zi})$ and those of the polygonal circuit (Zij). 


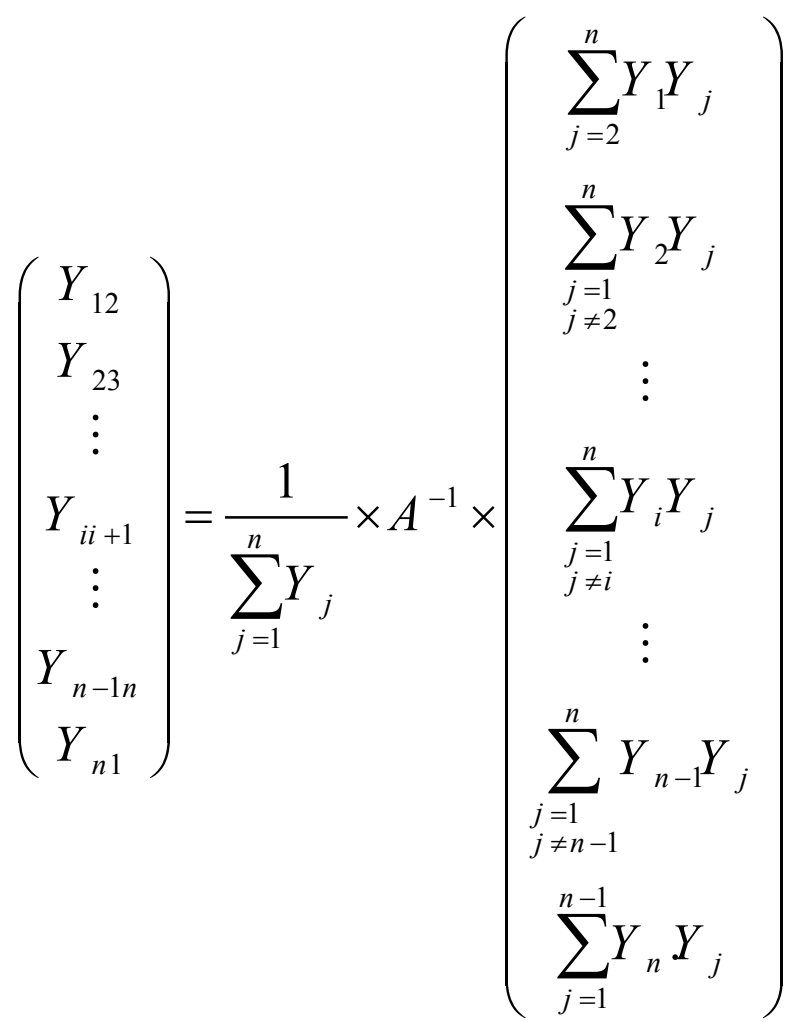

$$
\left(\begin{array}{c}
Z_{1} \\
Z_{2} \\
\vdots \\
Z_{i} \\
\vdots \\
Z_{n-1} \\
Z_{n}
\end{array}\right)=\frac{Z_{12}\left(\sum_{j=2}^{n-1} Z_{j j+1}+Z_{n 1}\right)}{Z_{23}\left(Z_{12}+\sum_{j=3}^{n-1} Z_{j j+1}+Z_{n 1}\right)}\left(\begin{array}{c}
Z_{j j+1}+Z_{n 1} \\
\vdots
\end{array} \times B^{-1} \times\left(\begin{array}{c}
Z_{i i+1}\left(\sum_{j=1}^{i-1} Z_{j j+1}+\sum_{j=i+1}^{n-1} Z_{j j+1}+Z_{n 1}\right. \\
\vdots \\
Z_{n-1 n}\left(\sum_{j=1}^{n-2} Z_{j j+1}+Z_{n 1}\right) \\
Z_{n 1}\left(\sum_{j=1}^{n-1} Z_{j j+1}\right.
\end{array}\right)\right.
$$


Where:

$$
\begin{aligned}
A & =\left(\begin{array}{cccccccc}
1 & 0 & \cdots & \cdots & \cdots & \cdots & 0 & 1 \\
1 & 1 & 0 & \vdots & \vdots & \vdots & \vdots & 0 \\
0 & 1 & 1 & 0 & \vdots & \vdots & \vdots & \vdots \\
\vdots & 0 & 1 & 1 & 0 & \vdots & \vdots & \vdots \\
\vdots & \vdots & 0 & \ddots & \ddots & \ddots & \vdots & \vdots \\
\vdots & \vdots & \vdots & \ddots & \ddots & \ddots & \ddots & \vdots \\
\vdots & \vdots & \vdots & \vdots & \ddots & \ddots & \ddots & 0 \\
0 & \cdots & \cdots & \cdots & \cdots & 0 & 1 & 1 \\
& & & & & & & \\
& & & & & & &
\end{array}\right) \\
& =\left(\begin{array}{cccccccc}
1 & 1 & 0 & \cdots & \cdots & \cdots & \cdots & 0 \\
0 & 1 & 1 & \vdots & \vdots & \vdots & \vdots & 0 \\
\vdots & 0 & 1 & 1 & \vdots & \vdots & \vdots & \vdots \\
\vdots & \vdots & 0 & 1 & 1 & \vdots & \vdots & \vdots \\
\vdots & \vdots & \vdots & \ddots & \ddots & \ddots & \vdots & \vdots \\
\vdots & \vdots & \vdots & \vdots & \ddots & \ddots & \ddots & \vdots \\
0 & \vdots & \vdots & \vdots & \vdots & \ddots & \ddots & 1 \\
1 & 0 & \cdots & \cdots & \cdots & \cdots & 0 & 1 \\
& & & & & & &
\end{array}\right)
\end{aligned}
$$

In order to prove the possibility of transforming a polygonal circuit into a star circuit or the inverse, we proceed with a demonstration of this generalized theorem in the following paragraph.

\section{Demonstration and Proof}

To demonstrate the validity of the polygon-star transformation or the inverse, it is necessary to find the circuit polygon elements according to the star circuit elements. This transformation is represented by (7) and (8).

To demonstrate (7) we apply the superposition theorem, putting the potential of all the nodes of both circuits, except the kth node, to the ground. This procedure takes us to the circuits of figures (see (figure 5) and (figure 6)).



Figure 5. Equivalent of the polygon network

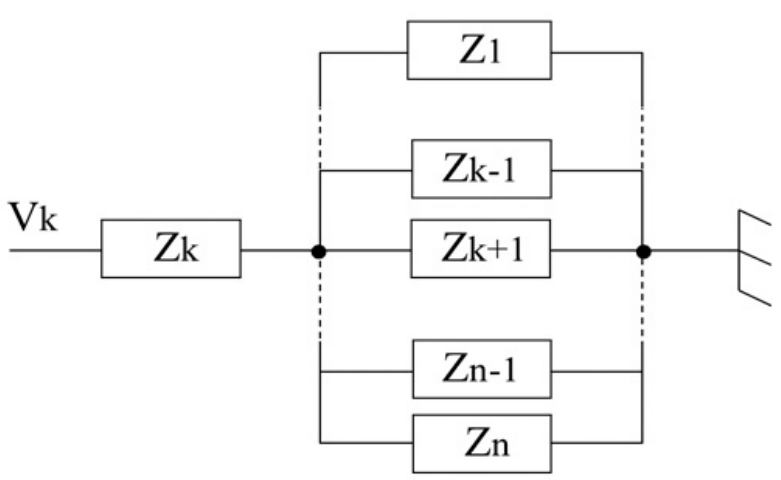

Figure 6. Equivalent of the star network

$$
\text { for } \mathrm{k}=1: Y_{n 1}+Y_{12}=\frac{\sum_{i=2}^{n} Y_{1} Y_{i}}{\sum_{i=1}^{n} Y_{i}}
$$

for $\mathrm{k} \in[|2, \mathrm{n}-1|]: Y_{k-1 k}+Y_{k k+1}=\frac{\sum_{i=1 ; i \neq k}^{n} Y_{k} Y_{i}}{\sum_{i=1}^{n} Y_{i}}$

$$
\text { for } \mathrm{k}=\mathrm{n}: Y_{n-1 n}+Y_{n 1}=\frac{\sum_{i=1}^{n-1} Y_{1} Y_{i}}{\sum_{i=1}^{n} Y_{i}}
$$

By gathering equations 11, 12 and 13 in matrix form, we get the following equation: 


$$
A \times\left(\begin{array}{c}
Y_{12} \\
Y_{23} \\
\vdots \\
Y_{i i+1} \\
\vdots \\
Y_{n-1 n} \\
Y_{n 1}
\end{array}\right)=\frac{1}{\sum_{j=1}^{n} Y_{j}} \times\left(\begin{array}{c}
\sum_{j=2}^{n} Y_{1} Y_{j} \\
\sum_{\substack{j=1 \\
j \neq 2}}^{n} Y_{2} Y_{j} \\
\vdots \\
\sum_{\substack{j=1 \\
j \neq i}}^{n} Y_{i} Y_{j} \\
\vdots \\
\sum_{\substack{j=1 \\
j \neq n-1}}^{n} Y_{n-1} Y_{j} \\
\sum_{j=1}^{n-1} Y_{n} Y_{j}
\end{array}\right)
$$

To find the equation (8), the matrix A must be invertible, so:

$$
\operatorname{det}(A)=1+(-1)^{n+1}
$$

The matrix A is invertible because $\mathrm{n}$ is odd. (This is the condition that we set at the beginning).

To demonstrate (8), we disconnect all nodes except the $\mathrm{kth}$ and the $(\mathrm{k}+1)$ th. Whereas for $\mathrm{k}=\mathrm{n}$ we disconnect the nth and the first node. Figures (see (figure 7) and (figure 8)) show the equivalent of the two networks for $k \in[2, n-2]$.

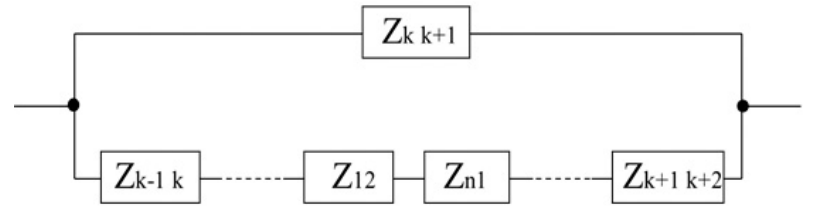

Figure 7. Equivalent of the polygon network for $\mathrm{k} \in[2, \mathrm{n}-2 \mid]$

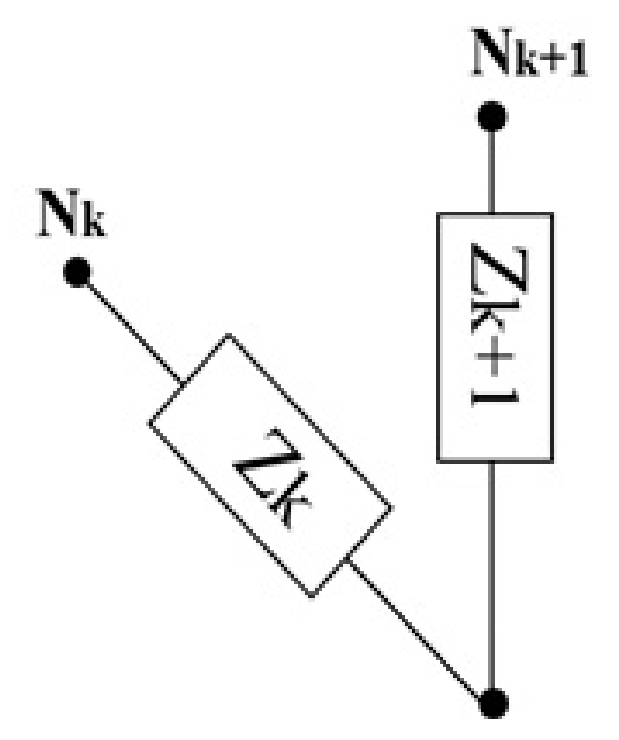

Figure 8. Equivalent of the star network for $k \in[2, n-2 \mid]$

From the two previous figures, we can deduce the following relations:

for $\mathrm{k} \in[2, \mathrm{n}-1]$ :

$$
Z_{k}+Z_{k+1}=\frac{Z_{k k+1}\left(\sum_{j=1}^{k-1} Z_{j j+1}+\sum_{j=k+1}^{n-1} Z_{j j+1}+Z_{n 1}\right)}{\sum_{j=1}^{n-1} Z_{j j+1}+Z_{n 1}}
$$

for $\mathrm{k}=\mathrm{n}$ :

$$
Z_{n}+Z_{1}=\frac{Z_{n 1}\left(\sum_{j=1}^{n-1} Z_{j j+1}\right)}{\sum_{j=1}^{n-1} Z_{j j+1}+Z_{n 1}}
$$

Based on (16) and (17) we come to the following equation in matrix form. 


$$
B \times\left(\begin{array}{c}
Z_{1} \\
Z_{2} \\
\vdots \\
Z_{i} \\
\vdots \\
Z_{n-1} \\
Z_{n}
\end{array}\right)=\frac{Z_{12}\left(\sum_{j=2}^{n-1} Z_{j j+1}+Z_{n 1}\right)}{\sum_{j=1}^{n-1} Z_{j j+1}+Z_{n 1}} \times\left(\begin{array}{c}
\left.Z_{12}+\sum_{j=3}^{n-1} Z_{j j+1}+Z_{n 1}\right) \\
\vdots \\
Z_{i i+1}\left(\sum_{j=1}^{i-1} Z_{j j+1}+\sum_{j=i+1}^{n-1} Z_{j j+1}+Z_{n 1}\right) \\
\vdots \\
Z_{n-1 n}\left(\sum_{j=1}^{n-2} Z_{j j+1}+Z_{n 1}\right) \\
Z_{n 1}\left(\sum_{j=1}^{n-1} Z_{j j+1}\right)
\end{array}\right)
$$

To find the expression of equation (8), matrix B must be invertible, so:

$$
\operatorname{det}(B)=1+(-1)^{n+1}
$$

The matrix B is invertible if and only if $\mathrm{n}$ is odd.

\section{Examples}

In this section, we apply the above theorem to two examples. The first is a network of three nodes as shown in (figure 1) and (figure 2). While the second is a balanced network of five nodes.

\subsection{Example 1}

In the case of a three-node network, we have:

$$
A^{-1}=\frac{1}{2} \times\left(\begin{array}{ccc}
1 & 1 & -1 \\
-1 & 1 & 1 \\
1 & -1 & 1
\end{array}\right) \text { and } B^{-1}=\frac{1}{2} \times\left(\begin{array}{ccc}
1 & -1 & 1 \\
1 & 1 & -1 \\
-1 & 1 & 1
\end{array}\right) \text {. }
$$

Equations (7) and (8) become:

$$
\begin{aligned}
& \left(\begin{array}{l}
Y_{12} \\
Y_{23} \\
Y_{31}
\end{array}\right)=\frac{1}{2 \sum_{j=1}^{3} Y_{j}} \times\left(\begin{array}{ccc}
1 & 1 & -1 \\
-1 & 1 & 1 \\
1 & -1 & 1
\end{array}\right) \times\left(\begin{array}{l}
Y_{1} Y_{2}+Y_{1} Y_{3} \\
Y_{2} Y_{1}+Y_{2} Y_{3} \\
Y_{3} Y_{1}+Y_{3} Y_{2}
\end{array}\right) \\
& \left(\begin{array}{l}
Z_{1} \\
Z_{2} \\
Z_{3}
\end{array}\right)=\frac{1}{2\left(Z_{12}+Z_{23}+Z_{31}\right)} \times\left(\begin{array}{ccc}
1 & -1 & 1 \\
1 & 1 & -1 \\
-1 & 1 & 1
\end{array}\right) \times\left(\begin{array}{l}
Z_{12}\left(Z_{23}+Z_{31}\right) \\
Z_{23}\left(Z_{12}+Z_{31}\right) \\
Z_{31}\left(Z_{23}+Z_{12}\right)
\end{array}\right)
\end{aligned}
$$

The simplification of (20) and (21) gives the same results of section 2. 


\subsection{Example 2}

Let us consider the five-node balanced networks depicted in (figure 9) and (figure 10).

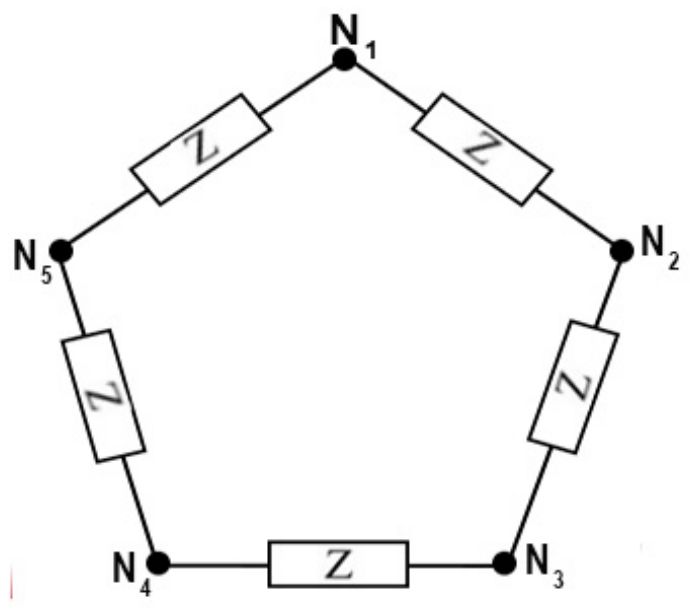

Figure 9. The five-node balanced network in polygonal form

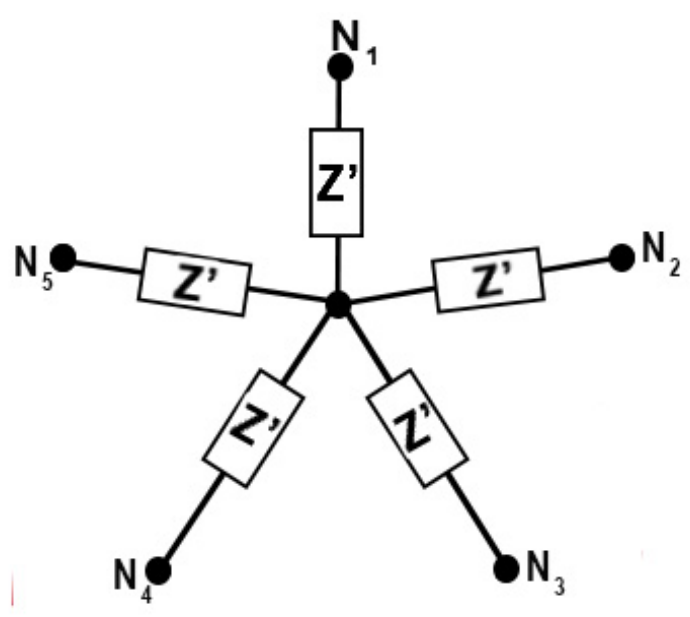

Figure 10. The five-node balanced network in star form

Firstly, we compute the inverse of matrices A and B:

$$
\begin{aligned}
A^{-1} & =\frac{1}{2} \times\left(\begin{array}{ccccc}
1 & 1 & -1 & 1 & -1 \\
-1 & 1 & 1 & -1 & 1 \\
1 & -1 & 1 & 1 & -1 \\
-1 & 1 & -1 & 1 & 1 \\
1 & -1 & 1 & -1 & 1
\end{array}\right) \text { and } \\
B^{-1} & =\frac{1}{2} \times\left(\begin{array}{ccccc}
1 & -1 & 1 & -1 & 1 \\
1 & 1 & -1 & 1 & -1 \\
-1 & 1 & 1 & -1 & 1 \\
1 & -1 & 1 & 1 & -1 \\
-1 & 1 & -1 & 1 & 1
\end{array}\right)
\end{aligned}
$$

Then, the simplification of (7) and (8) applied to this example yields:

$$
Z^{\prime}=\frac{2 Z}{5}
$$

So, the networks of (figure 9) and (figure 10) are equivalent if and only if (22) holds.

\section{Conclusions}

In this paper, we have presented a generalization of Kennelly's theorem, which can be used to reduce the complexity of some networks by transmuting the polygonal form to the star form and vice versa. First, the classical Kennelly's theorem has been presented. Then, the general theorem was introduced. After that, a demonstration based on the superposition theorem was proposed. Finally, two application examples were carried out to show the effectiveness of our theorem.

The results of this paper are very useful for educational purposes. This is due to the simplicity of the proposed formulation.

\section{REFERENCES}

[1] A. Starr, A new theorem for active networks, Journal of the institution of Electrical Engineers, Vol. 73, 303-308, Sept. 1933.

[2] G. Corazza, C. Someda, and G. Longo, Generalized Thevenin's Theorem for Linear N-Port Networks, IEEE Transactions On Circuit Theory, Vol.16, 564-566, Nov. 1969.

[3] J. Bird, Electrical Circuit Theory and Technology. Oxford, U.K.: Elsevier, 2003.

[4] F. Dorfler, J. W. Simpson-Porco, and F. Bullo, Electrical networks and Algebraic graph theory: Models, properties, and applications, Proceedings of the IEEE, Vol. 106, No. 5, 977-1005, May 2018.

[5] S. Y. Caliskan and P. Tabuada, "Towards kron reduction of generalized electrical networks," Automatica, Vol. 50, No. 10, 2586-2590, 2014.

[6] V. V. B. Rao and V. K. Aatre, Mesh-star transformation, Electron.Lett., Vol. 10, No. 6, 73-74, Mar. 1974.

[7] S. Bedrosian, Converse of the star-mesh transformation, IRE Trans. Circuit Theory, Vol. 8, No. 4, 491-493, Dec. 1961.

[8] D. W. C. Shew, XXVII. Generalized star and mesh transformations, London, Edinburgh, Dublin Phil. Mag. J. Sci., Vol. 38, No. 279, 267-275, 1947.

[9] L. Versfeld, Remarks on star-mesh transformation of electrical networks, Electron. Lett., Vol. 6, No. 19, 597-599, Sep. 1970.

[10] C. Wang and Y. Tokad, Polygon to star transformations, IRE Trans. Circuit Theory, Vol. CT-8, No. 4, 489-491, Dec. 1961. 